\title{
A Passive Stewart Platform Based Joystick to Control Spatially Moving Objects
}

\author{
Ibrahim YILDIZ \\ Mechatronics Engineering, Faculty of Engineering and Architecture, Necmettin Erbakan University, Konya 42090, Turkey
}

\begin{abstract}
Most of the spatially moving vehicles and game controllers use a 2-3 DOF (degrees of freedom) joystick to manipulate objects position. However, most of the spatially moving vehicles have more than 3 degrees of freedom, such as helicopters, quadrotors, and planes. Therefore, additional equipment like pedals or buttons is required during the manipulation. In this study, a passive Stewart platform based 6 degrees of freedom joystick was developed to control spatially moving objects. The Stewart platform mechanism is a 6-degrees of freedom parallel mechanism, which has been used for simulators. The main challenge of using a parallel mechanism to manipulate objects is the computational burden of its forward kinematics. Therefore, an artificial neural network was used for the forward kinematic solution of the Stewart platform mechanism to obtain the fastest response. Linear potentiometers were used for the Stewart platform legs. A mathematical model of a quadrotor was used to test the capability of the joystick. The developed spatial joystick successfully manipulated the virtual quadrotor model.
\end{abstract}

Key words: Spatial joystick, Stewart platform, artificial neural network, quadrotor.

\section{Introduction}

The purpose of this study is to emphasize the difficulty of traditional manipulation techniques, which are using a multi-point control such as a lever, pedals, and collective lever. This multi-tasking event is obviously difficult considering learning, teaching and application procedure. Therefore, the main objective of this study is gathering control tools of any SMV (spatially moving vehicles) into one hand. The advantages of one-handed manipulation are investigated with several scenarios, including reflection of external forces to SMV to the user's hand and force feedback control of manipulation mechanism. In this research, a linear potentiometer based SP (Stewart platform) as a man-machine interface is proposed as a means of mechatronic design, kinematic analysis, and simulation environment to manipulate SMVs. Through the paper, first, the analysis of SP

Corresponding author: Ibrahim YILDIZ, assistant professor, Ph.D., research fields: robotics, rehabilitation robotics, system dynamics and simulation, machine dynamics, automatic control, force control and bilateral teleoperation, biomechanics, bond-graph modeling, microcontrollers, man-machine interfaces. mechanism for kinematics and design of the system is investigated. Then, a "Quadrotor" is modeled using the Newton-Euler approach, a PID (proportional integral derivative) controller is applied to the derived model and a user interface is constructed for operating both the Quadrotor model and SPM (Stewart platform mechanism) simultaneously. Lastly, the virtual Quadrotor model is manipulated with a real Stewart Platform Joystick.

Stewart-Gough Platform is a parallel mechanism which was first used as an universal tire test machine and a flight simulator by V. E. Gough (1956) and D. Stewart (1965), connecting a stationary lower and a mobile upper platform to the two ends of six actuators in parallel and obtaining three translational and three rotational DOF (degrees of freedom) in space $[1,2]$.

Many methods were studied for the solution of the kinematics of the SPM. A major piece of work on solving the forward kinematics of SPM was using the Bezout method [3]. This method allows deriving the kinematics from a 16th degree equation with one unknown variable. The fact that one needs a numerical solution to obtain the final equation is one of the 
disadvantages of the method. Obtaining the answer from 16 solutions is one of the disadvantages of this method. Another solution method uses Newton-Rhapson algorithm and this was presented for solving the forward kinematics, iteratively [4]. This method usually converges to the solution. However, if one of the encoder data is not clear or wrong, the solution is not able to converge. A nonlinear observer was designed for predicting the 3 rotational and 3 translational displacements of the SPM by using the state variables of the mechanism [5]. Artificial Neural Networks are also used for solving the forward kinematics of the SPM $[6,7]$. This method is the best suitable solution for real-time mechanisms.

Human and robot interaction introduces new control requirements over time [21]. One of them is the force control. Investigations and comparisons were made on many force control method ranging from the simplest to the most complex [8]. An active stiffness control, which is one of the force control methods, was applied with the help of a program for controlling 3 translational and 3 rotational axes [9]. A pneumatically actuated $6 \times 6 \mathrm{SPM}$ was developed for endoscopic surgery. The relationship between the endoscope and the surface was provided by a force control algorithm [10]. Force control and tactile control methods, which are very important in terms of human-machine interaction, are frequently used in rehabilitation systems. $6 \times 6$ SPM was designed for the rehabilitation of the ankle with a user interface [11]. A 3 -axis parallel robot was developed for the rehabilitation of the wrist and system performance was investigated including the therapist effect $[12,13]$. A $3 \times 3$ Stewart platform manipulator has been proposed to manipulate spatially moving vehicles with force feedback [18]. This mechanism has six linear actuators and a force/torque sensor to sense the applied force and it successfully manipulated spatially moving vehicles. Impedance force control method [19] has been applied to the manipulator. However, despite its small size, it is still too heavy for practical applications.

UAV (unmanned air vehicles) have become a center of attraction due to the contributions of the military, rescue and aerospace technologies. An important part of research and development activities is the 4-rotor aircraft quadrotor, which has 4 DOF and motion capability at 6 axes. The Newton-Euler method has been used in many studies for obtaining the mathematical model of the quadrotor [14, 15]. Traditional and robust control algorithms were also applied to a quadrotor [15-17]. A simplified deterministic model of a quadrotor was presented for investigating the problem of planning/replanning [20]. In general, quadrotors and other aerial vehicles have multiple manipulation points. The main disadvantage of the developed manipulation mechanism is that the user must carry out multiple tasks at the same time in a standard multi-point manipulation. Briefly, the contribution of this work is the designed mechanism that allows the user to control all tasks from a single point. Section 2.1 describes the mechatronic design of the passive Stewart platform mechanism. Section 2.2 presents the forward kinematic solution of the mechanism. Section 2.3 describes the user interface of the software and the specifications of the mathematical model of the quadrotor. Section 3 consists of the experimental results and discussions. Section 4 is the conclusion section and addresses future works.

\section{Materials and Methods}

\subsection{Mechatronic Design and Specifications}

The legs of the SP consist of six linear potentiometers. The resistance of these potentiometers varies from 0 to $5 \mathrm{k} \Omega$ with the range of 0 to $100 \mathrm{~mm}$. The diameter of the upper ring (mobile ring) of the SP is $100 \mathrm{~mm}$. The diameter of the lower ring (stationary ring) is $140 \mathrm{~mm}$.

Potentiometers were connected both to the upper and lower part of the SP with spherical joints. Analog input ports of an Atmega powered Arduino Uno board were used both to obtain voltage values from potentiometers and to send position and orientation values of the SP to the computer, which are obtained from forward 
kinematics. The experimental setup of the SP joystick can be seen in Fig. 1.

\subsection{Forward Kinematics}

Obtaining position and orientation of the mobile ring of the SP mechanism from the leg lengths can be called forward kinematics solution. Iterative solutions and optimized solutions to solve the forward kinematics of the SP mechanism have been proposed. However, iterative solutions are not suitable for real-time applications because of time delay. In this study, ANN (artificial neural networks) are used to solve the forward kinematics of the SP which is suitable for real-time applications because of fast response. First of all, the workspace of the SP mechanism is scanned with inverse kinematics to obtain training data, which are the leg lengths for inputs and position/orientation values for targets of ANN. Lawenberg \& Marquard feed forward back propagation algorithm is used to train ANN. The architecture of the network can be seen in Fig. 2. Weight and bias values of the network are embedded in the controller board after training of the network. 20 neurons are used inside the hidden layer. The leg lengths of the SP, which are obtained from the potentiometer data, are the inputs to the network, as seen in Eq. (1).

$$
L=\left[\begin{array}{llllll}
L_{1} & L_{2} & L_{3} & L_{4} & L_{5} & L_{6}
\end{array}\right]
$$

The output of the hidden layer can be expressed as using Eq. (2) where " $L$ " is the leg length vector from Eq. (1), "W" and "B" are the weight and bias vectors obtained from the trained network, respectively. The weight value is the gain of an input of a neuron. This value changes over the training period of the neuron considering the importance of the input. As an example, if the effect of an input is important for the output, the value of the weight of the neuron should be bigger.

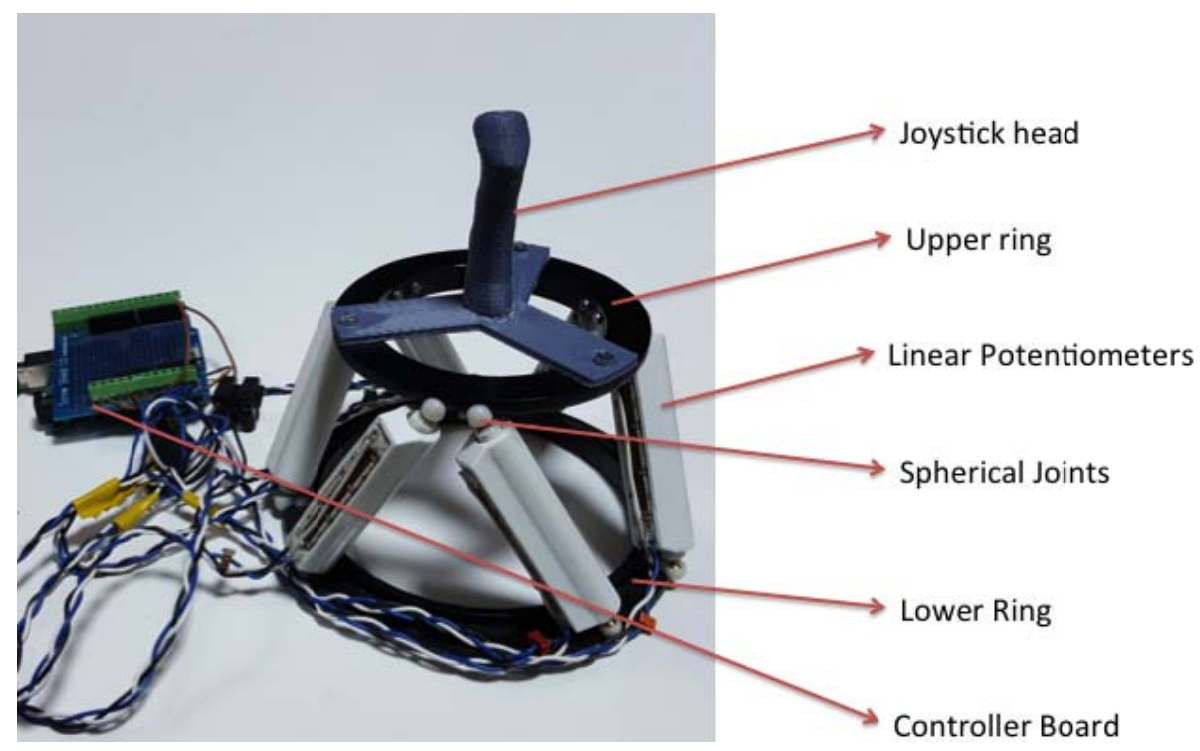

Fig. 1 Experimental setup of the SP joystick.

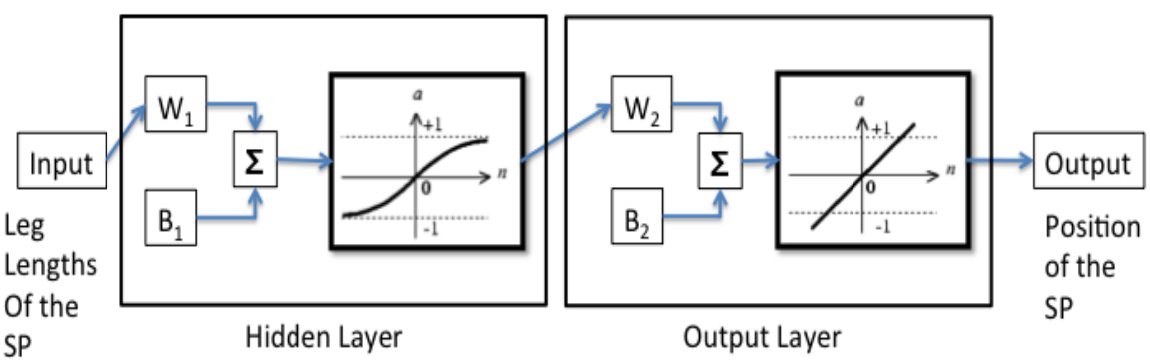

Fig. 2 Artificial neural network architecture of the forward kinematics of the SP. 
The bias value is the minimum stimulation level of the neuron. The sensitivity of the neuron is inversely proportional to the bias value. Mentioned ANN coefficients are decided during the learning process. The output of a neuron could be a linear or nonlinear function of input values. In the present study, tangent sigmoid function (a special case of logistic function) is used for the neurons in the hidden layer and linear function is used for the neurons in the output layer. The output of the first layer can be expressed as following.

$$
\text { Out }_{1}=2 / 1+\left(e^{-2\left(L \cdot W_{1}+B_{1}\right)}\right)
$$

The output of the complete ANN gives the position and orientation vector of the SP as in Eq. (3).

$$
\text { Output }=\left(2 / 1+\left(e^{-2\left(L \cdot W_{1}+B_{1}\right)}\right)\right) \cdot W_{2}+B_{2}
$$

\subsection{Computer Interface}

The communication between the SP joystick and the computer was provided with an rs 232 communication protocol [22]. A test program was written which includes the dynamic model of a quadrotor. The controller board of the SP joystick sends the position and orientation data to the computer. These outputs of the SP joystick are the reference inputs of the quadrotor model. A quadrotor mechanism has 6 degrees of freedom where two of them are dependent on angular motion around its $\mathrm{x}$ - and $\mathrm{y}$-axes. That means, if a quadrotor needs to move along $\mathrm{x}$ - or $\mathrm{y}$-axes, it must rotate itself around these axes.

Dynamic model and control strategies of a quadrotor have been investigated by many of researchers. In this study, the dynamic model of the quadrotor is constructed by using the Newton-Euler approach [14, 15]. A schematic representation of a quadrotor can be shown in Fig. 3. $\mathbf{F}_{1-4}$ represents the thrust generated by the propellers, $\ell$ is the distance of the motors to the center of mass of the quadrotor, capital $\mathrm{X}, \mathrm{Y}, \mathrm{Z}$ is the translational displacement of the quadrotor, $\phi^{q}, \theta^{q}, \psi^{q}$ are the rotational displacements of the quadrotor along the x, $\mathrm{y}, \mathrm{z}$-axes, $M_{t}$ is the mass of the quadrotor, $g$ is the acceleration due to gravity.

A quadrotor contains $4 \mathrm{dc}$ motor actuated propellers, which are mounted on the body. Each propeller generates reverse torques onto the body of the quadrotor. The rotation directions of propellers are selected to eliminate this effect.

Spatially moving vehicles are exposed to various physical effects like aerodynamics, inertia, gravity, gyroscopic forces and friction. Some of the assumptions listed below were used for modeling.

- Ground effect is neglected;

- Propeller blades are not flexible;

- Construction of mechanism is rigid and symmetric;

- Thrust and drag forces are proportional to the square of propeller velocities;

- Air drag friction is included.

The dynamics of the quadrotor body under external forces and torques can be expressed by Newton-Euler approach:

$$
\left[\begin{array}{cc}
M_{t} \cdot I_{b} & 0 \\
0 & J_{q}
\end{array}\right]\left[\begin{array}{c}
\dot{\vec{V}}^{q} \\
\dot{\vec{\omega}}^{q}
\end{array}\right]+\left[\begin{array}{c}
\vec{\omega}^{q} \times M_{t} \cdot \vec{V}^{q} \\
\vec{\omega}^{q} \times J_{q} \cdot \vec{\omega}^{q}
\end{array}\right]=\left[\begin{array}{c}
\vec{F}^{q} \\
\vec{\tau}^{q}
\end{array}\right]
$$

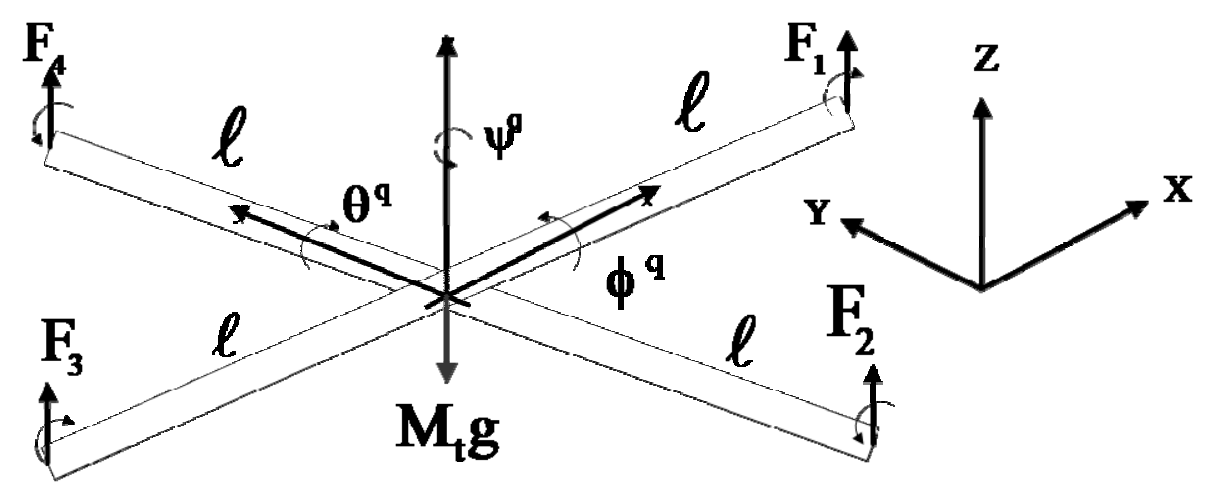

Fig. 3 Schematic representation of a quadrotor. 
Where; $I_{b}$ is $3 \times 3$ identity matrix, $J_{q}$ is the $3 \times 3$ inertia matrix of quadrotor, $\vec{V}^{q}$ is the linear velocity vector of the quadrotor, $\vec{\omega}^{q}$ is the angular velocity vector of the quadrotor, $\vec{F}^{q}$ is the total force vector of the quadrotor, $\overrightarrow{\boldsymbol{\tau}}^{q}$ is the total torque vector of the quadrotor.

Equations of motion of quadrotor model can be calculated using Eq. (4).

$$
\begin{gathered}
\dot{v}_{x}=\left(C \psi^{q} S \theta^{q} C \varphi^{q}+S \psi^{q} S \varphi^{q}\right) \cdot \sum_{1}^{4} \frac{b}{M_{t}} \Omega_{i}^{2}+\frac{F_{R x}^{q}}{M_{t}}+\frac{F_{h x}}{M_{t}}(5) \\
\dot{v}_{y}=\left(S \psi^{q} S \theta^{q} C \varphi^{q}-C \psi^{q} S \varphi^{q}\right) \cdot \sum_{1}^{4} \frac{b}{M_{t}} \Omega_{i}^{2}+\frac{F_{R y}^{q}}{M_{t}}+\frac{F_{h y}}{M_{t}}(6) \\
\dot{v}_{z}=\left(C \theta^{q} C \varphi^{q}\right) \cdot \sum_{1}^{4} \frac{b}{M_{t}} \Omega_{i}^{2}-g+\frac{F_{R z}^{q}}{M_{t}} \\
\ddot{\varphi}^{q}=\dot{\theta}^{q} \dot{\psi}^{q}\left(\frac{J_{y}-J_{z}}{J_{x}}\right)-\frac{J_{R}}{J_{x}} \dot{\theta}^{q} \Omega_{t}+\frac{\ell \cdot b}{J_{x}} \cdot\left(\Omega_{4}^{2}-\Omega_{2}^{2}\right) \\
\ddot{\theta}^{q}=\dot{\varphi}^{q} \dot{\psi}^{q}\left(\frac{J_{z}-J_{x}}{J_{y}}\right)+\frac{J_{R}}{J_{y}} \dot{\varphi}^{q} \Omega_{t}+\frac{\ell \cdot b}{J_{y}} \cdot\left(\Omega_{3}^{2}-\Omega_{1}^{2}\right) \\
\ddot{\psi}^{q}=\dot{\theta}^{q} \dot{\varphi}^{q}\left(\frac{J_{x}-J_{y}}{J_{z}}\right)+\frac{d}{J_{z}} \cdot\left(\Omega_{4}^{2}+\Omega_{2}^{2}-\Omega_{1}^{2}-\Omega_{3}^{2}\right)
\end{gathered}
$$

From the given equations of motion, thrust factor of the quadrotor is demonstrated by $b, d$ is the drag factor, $\Omega_{i}$ is the angular velocity of the propellers ( $i=1$ to 4$), F_{R(x, y, z)}^{q}$ is the external forces acting on quadrotor from the axes $\mathrm{x}, \mathrm{y}$, and $\mathrm{z}, F_{h(x, y, z)}$ is the force caused by air resistance from the axes $x, y$, and $z, \Omega_{t}$ is the total rotor velocities considering the direction of rotation, $v_{x, y, z}$ is the linear velocities of quadrotor.

For the purpose of the study, position of the quadrotor mechanism was intended to manipulate by the SP. Because of this reason, only 4 position controllers designed. Motion on axes $\varphi^{q}, \theta^{q}, \psi^{q}, z$ are directly related to control inputs. Therefore, propeller velocities must be including the control inputs on both 4 axes. Motion on axes $\mathrm{x}$ and $\mathrm{y}$ are related to the displacement on axes $\theta^{q}$ and $\varphi^{q}$, respectively. For instance, if there is an angular motion on axis $\varphi^{q}$, quadrotor moves along the y-axis. Therefore, 4 PID controllers designed for controlling the quadrotor mechanism:

$$
\begin{aligned}
& \Omega_{c z}=k p_{z} \cdot e_{z}+k d_{z} \cdot \dot{e}_{z}+k i_{z} \cdot \int e_{z} \\
& \Omega_{c \varphi}=k p_{\varphi} \cdot e_{\varphi}+k d_{\varphi} \cdot \dot{e}_{\varphi}+k i_{\varphi} \cdot \int e_{\varphi} \\
& \Omega_{c \theta}=k p_{\theta} \cdot e_{\theta}+k d_{\theta} \cdot \dot{e}_{\theta}+k i_{\theta} \cdot \int e_{\theta} \\
& \Omega_{c \psi}=k p_{\psi} \cdot e_{\psi}+k d_{\psi} \cdot \dot{e}_{\psi}+k i_{\psi} \cdot \int e_{\psi}
\end{aligned}
$$

Eqs. (11-14) are the contribution of controllers to the actual rotor velocities. Because of the geometric design of the system, each rotor velocity must be calculated by using related controllers. Controller coefficients $k p_{(z, \varphi, \theta, \psi)}, k d_{(z, \varphi, \theta, \psi)}, k i_{(z, \varphi, \theta, \psi)}$ are proportional, derivative and integral coefficients, respectively. Schematic representation of controllers can be seen in Fig. 4.

All motors have equal contribution on the motion along z-axis of the quadrotor. Therefore, Eq. (11) must be driving all rotor velocities. 2 nd and 4 th motors have contribution on the motion around $\varphi^{q}$. For this reason, Eq. (12) must be driving 2 nd and 4 th rotor velocities considering the direction of motion. Similarly, Eq. (13) must be added 1 st and 3rd rotor velocities because of the contribution on the motion around $\theta^{q}$. All rotors are effective on the motion of quadrotor around $\psi^{q}$. Therefore, Eq. (14) must be driving all rotor velocities as well. Final rotor velocities can be expressed as the given equation series below.

$$
\begin{aligned}
& \Omega_{1}=\Omega_{c z}-\Omega_{c \theta}-\Omega_{c \psi} \\
& \Omega_{2}=\Omega_{c z}-\Omega_{c \varphi}+\Omega_{c \psi} \\
& \Omega_{3}=\Omega_{c z}+\Omega_{c \theta}-\Omega_{c \psi} \\
& \Omega_{4}=\Omega_{c z}+\Omega_{c \varphi}+\Omega_{c \psi}
\end{aligned}
$$

The reference values of the controller were obtained from the output of the forward kinematics of the SP joystick. The position feedback of the quadrotor was obtained from the dynamic equations of the quadrotor. The PID coefficients of the controllers were decided after a series of simulation considering the settling time, overshoot and steady state error values.

The developed user interface of the test program can be seen in Fig. 5. The user interface allows the user to 


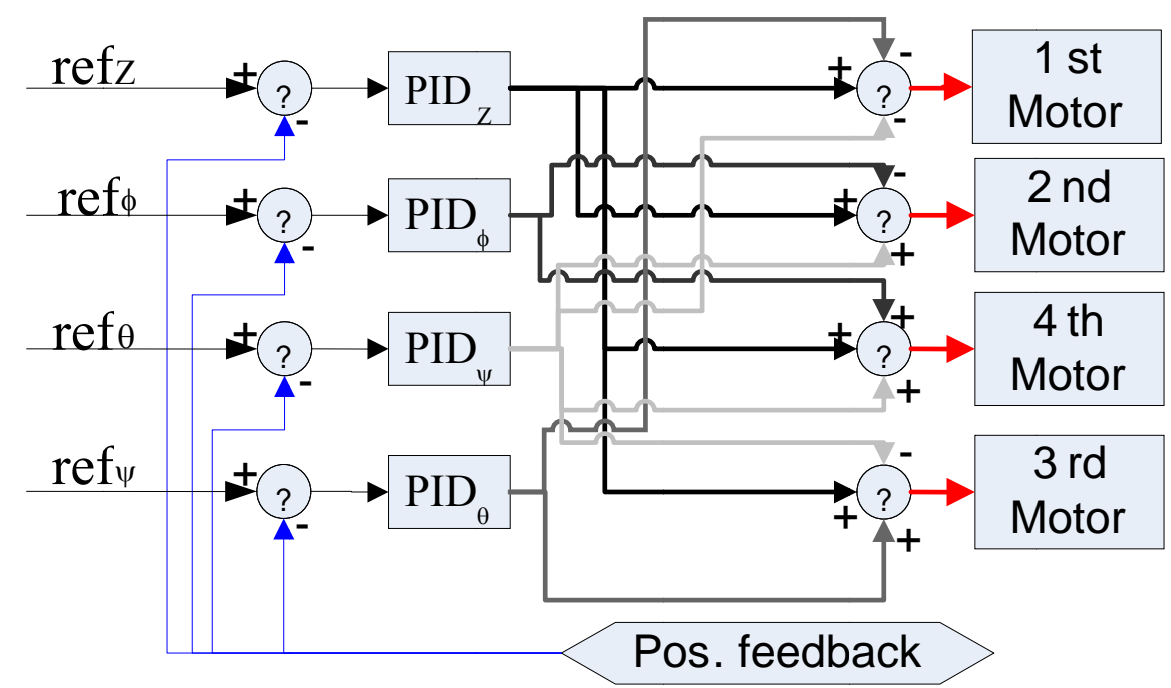

Fig. 4 Control schematic of the quadrotor model.

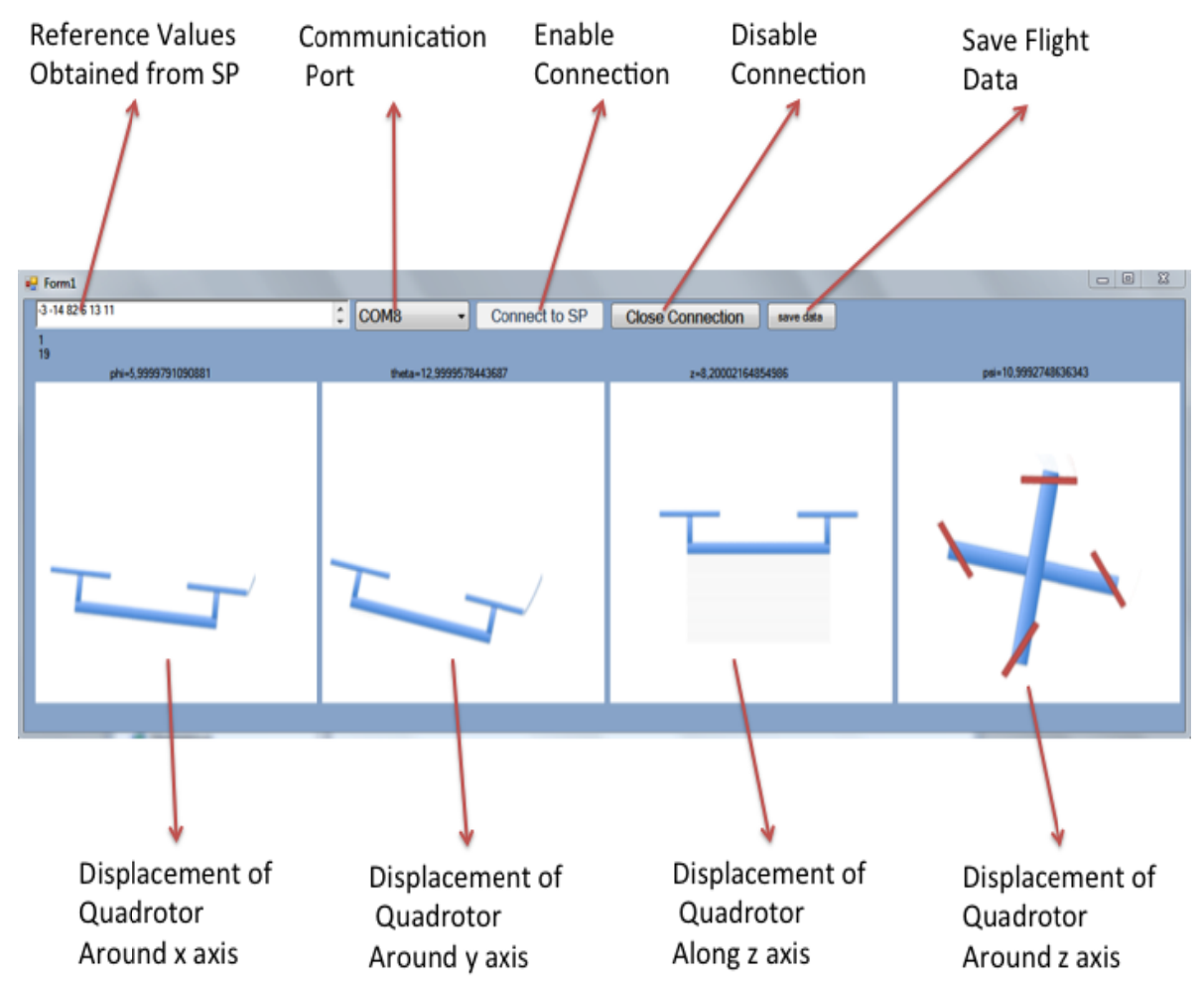

Fig. 5 User interface of the test program.

observe both the incoming data from the SP and the action of the quadrotor model considering the reference data coming from the SP joystick.

\section{Results and Discussions}

The dynamic model and the control of the quadrotor were tested with the developed interface. Reference values of $10 \mathrm{~m}$ for displacement along the $\mathrm{z}$-axis, 45 degrees for displacement around the z-axis, 15 degrees for displacement around the x-axis, -15 degrees for displacement around the $y$-axis were used. The response of the controller is shown in Fig. 6. The dynamic model of the quadrotor successfully reaches the reference values. 


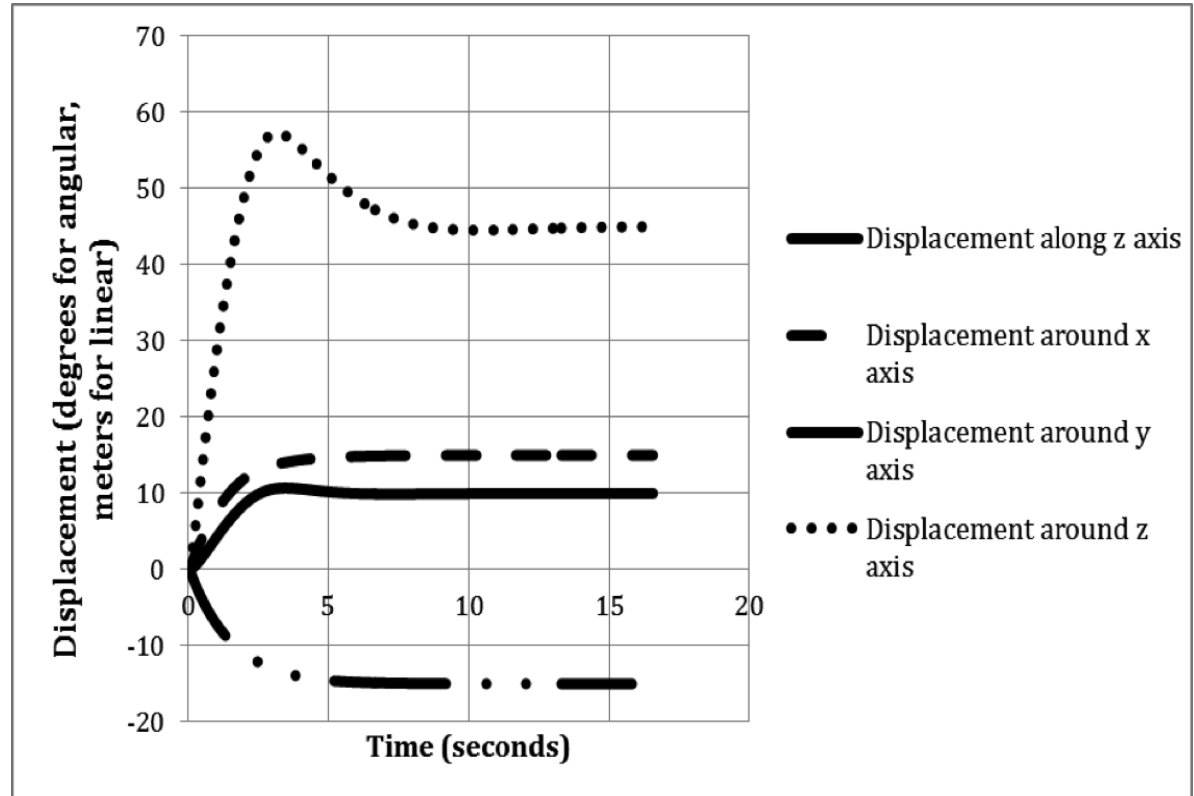

Fig. 6 Response of the PID controllers.

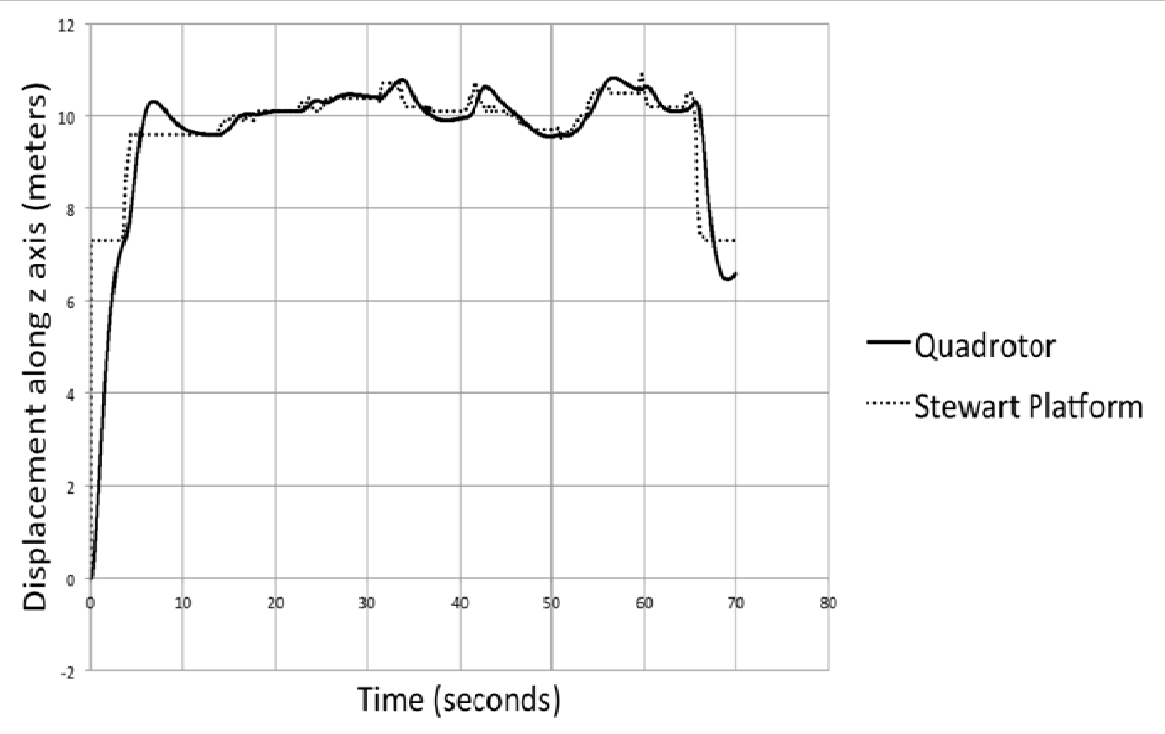

Fig. 7 Displacement of the quadrotor model and the SP joystick along the z-axis.

Manipulation of the dynamic quadrotor model was also tested with SP joystick. The reference values of the angular displacements of the quadrotor model are the displacement of the SP around the $\mathrm{x}-, \mathrm{y}-$ and z-axes. The reference value of the linear displacement of the quadrotor along the z-axis was the multiplication of displacement of the SP along the z-axis with a coefficient. One inexperienced user performed the test. Moving the quadrotor model separately around the x-, $\mathrm{y}$-, z-axis and along the z-axis was requested by the user.

The quadrotor successfully reaches the incoming reference values from the SP. Figs. 7-10 depict the displacement of both the quadrotor model and the SP joystick. The dynamics of the quadrotor and the PID controller cause a time delay between the reference values and the position of the quadrotor. This settling time of the quadrotor can be clearly seen in the mentioned figures. 


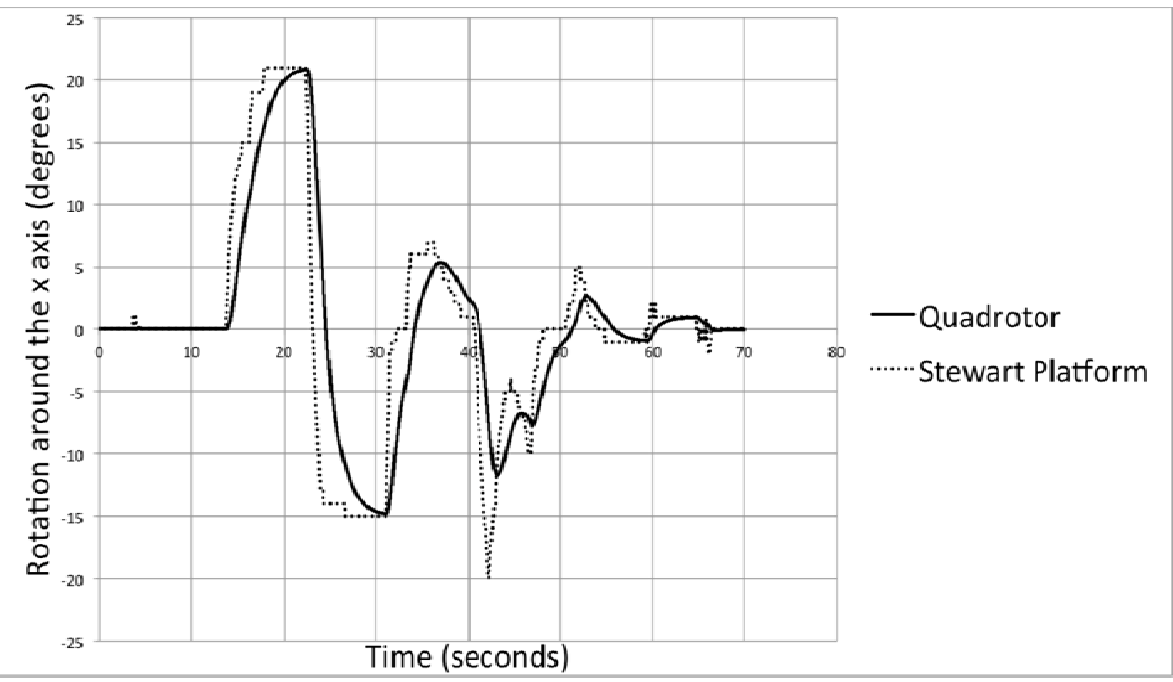

Fig. 8 The angular displacement of the quadrotor and the SP joystick around the $x$-axis.

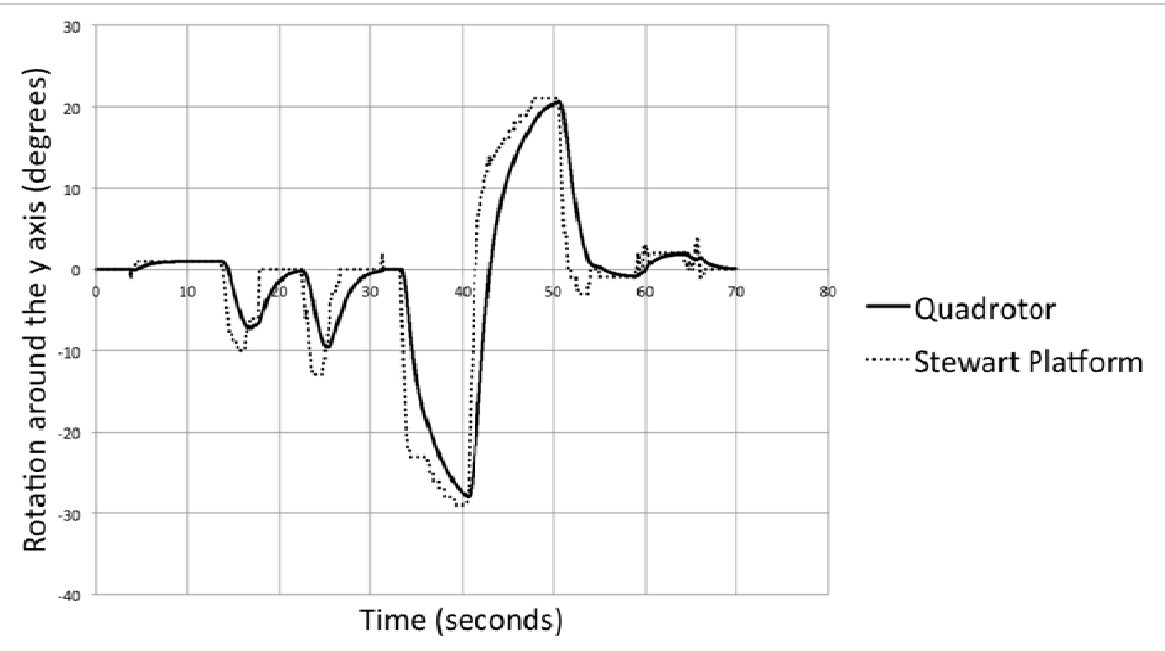

Fig. 9 The angular displacement of the quadrotor and the SP joystick around the y-axis.

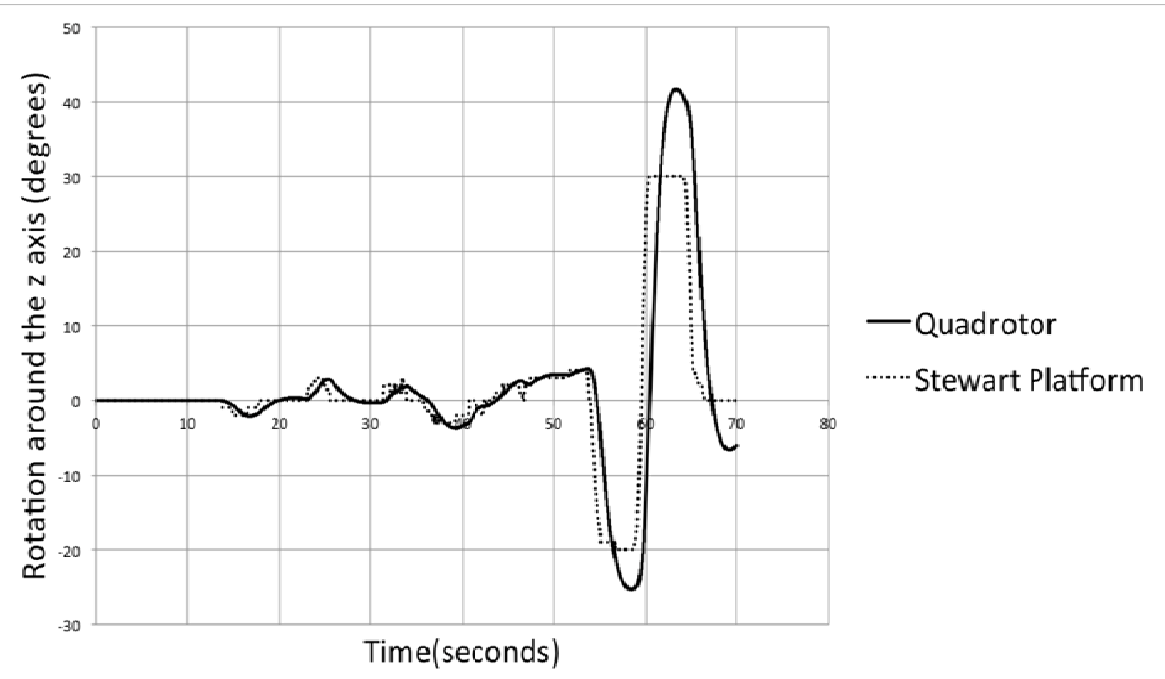

Fig. 10 The angular displacement of the quadrotor and the SP joystick around the z-axis. 


\section{Conclusions}

Manipulation of the spatially moving objects is important considering both the cost of the aerial vehicles and safety of passengers. Multiple manipulations might distract the user. The reason for many accidents is the distraction of the pilot or the user. Therefore, a passive 6 DOF Stewart platform based joystick was designed for single point manipulation. Classical iterative solutions of the forward kinematics of the SP cause time delays and errors considering real-time applications. Therefore, ANN was used for forward kinematics solution of the SP. A dynamic model of a quadrotor was derived for testing the manipulation of the SP joystick. PID controllers were used to control the axes of the quadrotor. A user interface was built containing the dynamic model of the quadrotor. Finally, the quadrotor model was successfully manipulated from a single point with the SP joystick.

It is very probable that the development of one point manipulation will become the center of attraction for most disciplines including simulation or game development. The developed low-cost Stewart platform joystick solution promises to open a new era for man-machine interface. Considering the future works, haptic feedback should be provided for the user to sense both the inertial and other external forces.

\section{Acknowledgement}

This work is supported by the Coordinatorship of Scientific Research Projects of Necmettin Erbakan University grant 162518001-056 and this work is also the extended version of the Ref. [23].

\section{References}

[1] Stewart, D. 1965. "A Platform with Six Degrees of Freedom." UK Institution of Mechanical Engineers Proceedings 180: 1-15.

[2] Gough, V. E. 1956. "Contribution to Discussion to Papers on Research in Automobile Stability and Control in Tyre Performance, by Cornell Staff." Proc. Auto Div. Inst. Mech. Eng.: 392-5.
[3] Nanua, P., Waldron, K. J., and Murthy, V. 1990. "Direct Kinematic Solution of a Stewart Platform." IEEE Transactions on Robotics and Automation 6: 438-44.

[4] Harib, K., and Srinivasan, K. 2003. "Kinematic and Dynamic Analysis of Stewart Platform Based Machine Tool Structures." Robotica 21: 541-54.

[5] Chen, S.-H., Huang, C.-I., and Fu, L.-C. 2008. "Applying a Nonlinear Observer to Solve Forward Kinematics of a Stewart Platform." IEEE International Conference on Control Applications: 1183-8.

[6] Yee, C. S., and Lim, K. 1997. "Forward Kinematics Solution of Stewart Platform Using Neural Networks." Neurocomputing 16: 333-49.

[7] Yurt, S. N., Anl1, E., and Ozkol, I. 2007. "Forward Kinematics Analysis of the 6-3 SPM by Using Neural Networks." Meccanica 42: 187-96.

[8] Zeng, G., and Hemami, A. 1997. "An Overview of Robot Force Control.” Robotica 15: 473-82.

[9] Salisbury, K. J. 1980. "Active Stiffness Control of a Manipulator in Cartesian Coordinates." The 19th IEEE Conf. on Decision and Control, 95-100.

[10] Benalli, A., Richard, P., and Bidaud, P. 1999. "Control and Evaluation of a Six DOF Force Feedback Interface for Virtual Reality Applications." Proceedings of the IEEE International Workshop on Robot and Human Interaction, 338-43.

[11] Girone, M., Burdea, G., Bouzit, M., and Popescu, V. 2001. "A Stewart Platform-Based System for Ankle Telerehabilitation." Autonomous Robots 10: 203-12.

[12] Gupta, A., O’Malley, M. K., Patoğlu, V., and Burgar, C. 2008. "Design, Control and Performance of Rice Wrist: A Force Feedback Wrist Exoskeleton for Rehabilitation and Training." The International Journal of Robotics Research 27 (2): 233-51.

[13] Satıc1, A. C., Erdoğan, A., and Patoğlu, V. 2009. "Design of a Reconfigurable Ankle Rehabilitation Robot and Its Use for the Estimation of the Ankle Impedance." IEEE 11th International Conference on Rehabilitation Robotics, 257-64.

[14] Bresciani, T. 2008. "Modelling, Identification and Control of a Quadrotor Helicopter." Master thesis, Department of Automatic Control, Lund University.

[15] Hamel, T., Mahony, R., Lozano, R., and Ostrowski, J. P. 2002. "Dynamic Modelling and Configuration Stabilization for a X4-Flyer." IFAC 15th World Congress on Automatic Control, 1.2: 3.

[16] Bouabdallah, S., and Siegwart, R. 2005. "Backstepping and Sliding-Mode Techniques Applied to an Indoor Micro Quadrotor." Proceedings of the IEEE, International Conference on Robotics and Automation, 2247-52.

[17] Bouabdallah, S., Noth, A., and Siegwart, R. 2004. "PID vs. LQ Control Techniques Applied to an Indoor 
Micro Quadrotor." Intelligent Robots and Systems 3: 2451-6.

[18] Ömürlü, V. E., and Yildiz, I. 2010. "Self-tuning Fuzzy PD-based Stiffness Controller of a 3x3 Stewart Platform as a Man-Machine Interface." Turkish Journal of Electrical Engineering and Computer Sciences, doi:10.3906/elk-1007-581.

[19] Hogan, N. 1985. "Impedance Control, an Approach to Manipulation: Part I, II.” Int. J. of Robotics Res. 107: 1-24.

[20] Chamseddine, A., Youmin, Z., Rabbath, C. A., Join, C., and Theilliol, D. 2012. "Flatness-Based Trajectory Planning/Replanning for a Quadrotor Unmanned Aerial Vehicle." IEEE Transactions on Aerospace and
Electronic Systems 4 (48): 2832-48.

[21] Salisbury, K. 1988. "Issues in Human/Computer Control of Dexterous Remote Hands." IEEE Transactions on Aerospace and Electronic Systems 24: 591-6.

[22] Electronics Industries Association et al. 1969. "EIA Standard RS-232-C Interface between Data Terminal Equipment and Data Communication Equipment Employing Serial Data Interchange." International Organization for Standardization, Geneva, Switzerland.

[23] Yildiz, I. 2016. "A PassiveStewart Platform Based Joystick to Control Spatially Moving Objects." ACHI 2016: The Ninth International Conference on Advances in Computer-Human Interactions, 79-83. 